\title{
Massive bone marrow necrosis and postnecrotic myelofibrosis in a patient with primary thrombocythaemia
}

\author{
G Majumdar, J K Phillips, T C Pearson
}

\begin{abstract}
A case of non-fatal massive bone marrow necrosis (MBMN) is reported in a patient with primary thrombocythaemia five years after diagnosis. No precipitating cause was identified. The patient developed postnecrotic myelofibrosis six months later. As far as is known, this is the first report of $M B M N$ in a case of primary thrombocythaemia.
\end{abstract}

(F Clin Pathol 1994;47:674-676)

Massive bone marrow necrosis (MBMN) is a rare and often terminal complication of both haematological and non-haematological malignancies, usually with bone marrow metastases, ${ }^{1}$ but it has also been reported in non-malignant conditions, such as sickle cell disease. $^{2}$ We report a case of non-fatal MBMN in a patient with primary thrombocythaemia, a complication which has not been reported before.

\section{Case report}

A 54 years old man presented with pain in both feet which he had had for six months, with discolouration of the toes for three weeks. He had no previous history of vasoocclusive disease. He smoked 20 cigarettes daily and drank very little alcohol. Clinically there was peripheral cyanosis of the toes but not of the fingers. All peripheral pulses were present and his blood pressure was 150/80 $\mathrm{mm} \mathrm{Hg}$. There was no pallor, jaundice, or lymphadenopathy. No atrophic changes were noted in the lower extremities. Systemic examination was normal; in particular, hepatosplenomegaly was absent. There was no evidence of any infection.

A full blood count showed a haemoglobin of $149 \mathrm{~g} / 1$, a white cell count of $11.3 \times 10^{9} / 1$, with a normal differential, and a platelet count of $1480 \times 10^{9} / 1$. Bone marrow aspirate showed substantially increased numbers of megakaryocytes but was otherwise normal. A bone marrow trephine biopsy specimen confirmed the increased number of megakaryocytes but no increase in reticulin. Serum ferritin was $60 \mu \mathrm{g} / 1$, red cell folate $237 \mu \mathrm{g} / 1$ and serum $B_{12}$ was $320 \mathrm{ng} / \mathrm{l}$. Liver function tests, urea and creatinine concentrations were normal. A chest $x$ ray picture did not show any abnormality. Platelet function tests were not done. Primary thrombocythaemia was diagnosed and he was treated with intermit- tent courses of busulphan $4 \mathrm{mg}$ daily for two weeks at a time. After three such courses over 10 weeks his blood picture was as follows: haemoglobin $139 \mathrm{~g} / \mathrm{l}$, white cell count $5.8 \times 10^{9} / 1$, and platelets at $311 \times 10^{\%} / 1$. He also had aspirin $150 \mathrm{mg}$ on alternate days for three months. His symptoms improved rapidly and the discolouration of the toes disappeared at the same time.

He continued to be in good health for the next five years and required three to four courses of low dose busulphan every year to keep the platelet count below $450 \times 10^{9} / 1$. His haemoglobin and white cell counts remained within the normal range and the platelet count varied between 320 and $450 \times 10^{9} / 1$. His blood count just before a holiday in Spain was: haemoglobin $142 \mathrm{~g} / \mathrm{l}$; white cell count of $7 \cdot 2 \times 10^{9} / 1$; and a platelet count of $391 \times 10^{9} / 1$. While on holiday, he felt unwell, with generalised pain, which was most severe in the ribs and back. He lost his appetite, felt feverish, and sweaty. He was given analgesics, with some relief of his symptoms. His condition improved gradually and the symptoms disappeared after about a week.

He did not seek medical advice during this period. Five weeks after this episode, by which time he was asymptomatic, his blood count showed a haemoglobin of $103 \mathrm{~g} / \mathrm{l}$, a white cell count of $5.6 \times 10^{9} / 1$, and a platelet count of $144 \times 10^{9} / 1$. No clinical abnormality was detected and he had no bony tenderness. A bone marrow aspirate yielded only peripheral blood and a trephine biopsy specimen showed MBMN but with no increase in reticulin (fig 1). Liver function tests were normal except for a raised serum alkaline phosphatase activity (163 IU/l). Viral antibody screening did not show any evidence of a recent viral infection. Platelet function tests were not done. The blood count improved slowly over the next six months, with haemoglobin reach-

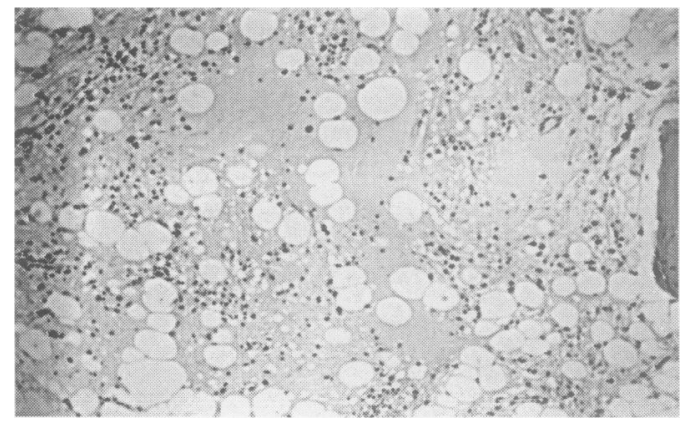

Figure 1 Bone marrow trephine biopsy specimen showing massive bone marrow necrosis without increased fibrosis (haematoxilin eosin). 
Figure 2 Bone marrow trephine biopsy specimen showing hypercellular marrow with substantial increase in coarse reticulin (reticulin stain).

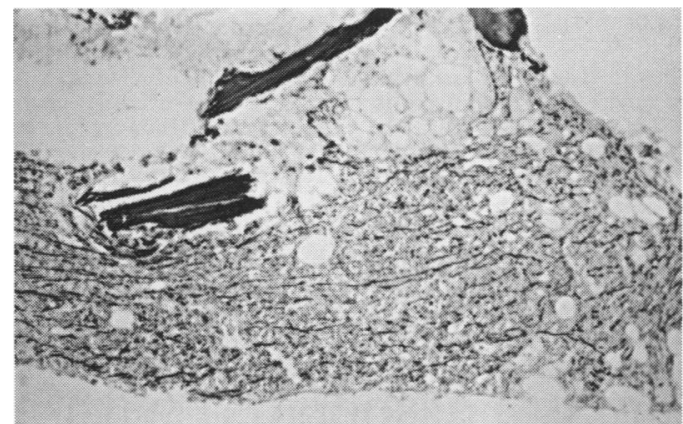

ing $122 \mathrm{~g} / \mathrm{l}$, white cell count $5.8 \times 10^{9} / 1$, and platelets at $283 \times 10^{9} / 1$. Subsequently his haemoglobin fell again to $108 \mathrm{~g} / 1$ and platelets to $210 \times 10^{9} / 1$, though the white cell count remained stable $\left(6.6 \times 10^{9} / 1\right)$. A blood film showed anisopoikilocytosis with tear drop cells, left shifted neutrophils together with a few metamyelocytes, and an occasional myelocyte. A repeat bone marrow aspiration failed but a trephine biopsy specimen showed increased cellularity with greatly increased coarse reticulin staining (fig 2 ). It was concluded that he had developed postnecrotic myelofibrosis. His blood count remains stable one year later, with no further busulphan treatment.

\section{Comments}

MBMN is a rare complication of haematological malignancies and has most often been described in patients with acute leukaemia. ${ }^{3}$ It has also been described in association with chronic granulocytic leukaemia with or without blast crisis, lymphomas, metastatic carcinoma, chemotherapy, alcohol abuse, HIV and other viral infections and in sickle cell crisis. ${ }^{124}$ It is often a terminal complication of non-haematological malignancy and is usually associated with bone marrow metastases. ${ }^{1} \mathrm{MBMN}$ has not been described before in association with primary thrombocythaemia.

MBMN is almost always associated with bony pain, particularly back pain, present in our patient. ${ }^{3}$ Other symptoms are usually related to the primary disease and not to the MBMN itself. Our patient had a substantial fall in haemoglobin and platelet count, recognised features of $\mathrm{MBMN} .{ }^{3}$ Both counts slowly improved over six months before stabilising at levels lower than those before the episode of MBMN. A raised alkaline phosphatase activity was noted in this patient, and this feature has also been reported in some other cases of MBMN. ${ }^{5}$ Interestingly, our patient has not required further marrow myelosuppressive treatment since the episode of MBMN. Bone marrow examination at this stage showed the features of myelofibrosis. Though myelofibrosis is a common complication of primary thrombocythaemia, the temporal relation with the $M B M N$ suggests that in this case it was a sequel to marrow necrosis.

The cause of $M B M N$ is not always apparent. Microcirculatory obstruction due to marrow hyperplasia, as in leukaemia, or marrow infiltration, as in metastatic carcinoma, has been suggested as the cause. ${ }^{1}$ However, MBMN is a widespread disease involving almost all active bone marrow sites and it is unlikely that microcirculatory obstruction would develop in multiple sites simultaneously. In such situations a chemical mediator is a more likely causative agent. Recently a high concentration of circulating tumour necrosis factor was found in two patients with $M B M N$ and this was considered to be the causative agent for the necrosis. ${ }^{6}$ Microvascular occlusion due to sickling crisis or disseminated intravascular coagulation is often widespread and may cause MBMN without any additional mediator.? Chemotherapy, both with single and multiple agents, had been held responsible for MBMN. ${ }^{89}$ In such cases bone marrow damage by chemotherapeutic agents could either be a direct effect or an indirect effect mediated through the release of various toxic substances from the dying cells. Cytomegalovirus infection was thought to be causally associated with $\mathrm{MBMN}$ in one case. ${ }^{10}$ However, in a small number of patients neither any underlying cause nor any precipitating factor could be identified. $^{2}$

The present case had a longstanding haematological disease which is associated with marrow hyperplasia, though his disease was apparently under good control when MBMN occurred. His platelet count was within the normal range for several months before the development of MBMN. However, thrombotic complications can occur in patients with primary thrombocythaemia without a high platelet count. This episode might have been related to chemotherapy, though low dose busulphan has never been reported as a causative agent for $M B M N$. Moreover, this patient had not received busulphan for 11 weeks before the development of MBMN. Therefore, chemotherapy was an unlikely cause for this episode. There was no history of alcohol abuse for our patient, even while on holiday, and no other precipitating factor, such as viral infection, could be identified.

The prognosis of $M B M N$ has been reported to be poor, with very few patients surviving for more than a few weeks. $^{1}$ Recently it has been recognised that the prognosis is related to the underlying disease and not to the MBMN itself which is often underdiagnosed. ${ }^{2}$ The outcome in our patient seems to be good compared with most of the reported cases of MBMN. However, he has probably developed permanent marrow changes as a result of this episode.

1 Scudla V, Dusek J, Macak J, Zivna J, Myslivecek M, Indrak $\mathbf{K}$. Bone marrow necrosis in malignant diseases. $A$ report on seven intravitally recognized cases. A report on seven intravi

2 Maisel D, Lim JY, Pollock WJ, Yatani R, Liu PI. Bone marrow necrosis: an entity often overlooked. Ann Clin marrow necrosis: an entity

3 Hansen PV, Andersen J, Mygind H. Bone marrow necrosis Report of a case and a brief review of the literature. Acta Med Scand 1983;214:331-6.

4 Norgard MJ, Carpenter JT Jr, Conrad ME. Bone marrow necrosis and degeneration. Arch Intern Med 1979;139:905-11

5 Vesterby A, Myhre Jensen O. Aseptic bone/bone marrow 
mecrosis in leukaemia, Scand $f$ Haematol 1985;35: 354-7.

6 Knupp C, Pekala PH, Cornelius P. Extensive bone marrow necrosis in patients with cancer and tumor necrosis factor activity in plasma. Am $f$ Hematol 1988;29:215-21.

7 Harigaya K, Watanabe S, Watanabe Y, Kageyama K Natakaya K. Multiple bone marrow necrosis and disseminated intravascular coagulation. Arch Pathol Lab Med 1977;101:652-4.
8 Brown $\mathrm{CH}$. Bone marrow necrosis. A study of seventy cases. fohns Hopkins Med $\mathcal{f}_{1972 ; 131: 189-203}$

9 Sporn JR, Fallon MA. Recovery from widespread bone marrow necrosis occurring after chemotherapy for adult marrow necrosis occurring after chemotherapy for adult acute monocy $1992 ; 20: 224-6$.

10 Rustgi VK, Sacher RA, O'Brien P, Garagusi VF. Fatal disseminated cytomegalovirus infection in an apparently normal adult. Arch Intern Med 1983;143:372-3.

\title{
Pure red cell aplasia associated with malignant thymoma, myasthenia gravis, polyclonal large granular lymphocytosis and clonal thymic $\mathrm{T}$ cell expansion
}

\author{
S I Handa, K P Schofield, M Sivakumaran, M Short, R S H Pumphrey
}

\begin{abstract}
A case with the triad of pure red cell aplasia (PRCA), myasthenia gravis, and malignant thymoma is reported. There was a clonal proliferation of $T$ cells within the thymoma, as demonstrated by a $T$ cell antigen receptor (TCR) $\delta$ chain gene rearrangement. However, despite a large granular lymphocytosis, clonality could not be shown in the peripheral blood either before or after thymectomy. There was no evidence of human $T$ cell lymphotrophic virus type 7 (HTLV1) infection.

It is postulated that the clonal thymic $T$ cell population secreted cytokine(s), which stimulated the polyclonal proliferation of large granular lymphocytes, which in turn suppressed erythropoiesis. Thymectomy removed the stimulus to the large granular lymphocytes and hence there was a resurgence of erythropoiesis.
\end{abstract}

Department of

Haematology, North

Manchester General

Hospital Manchester $S$ I Handa

K P Schofield

Department of

Haematology,

Leicester Royal

Infirmary, Leicester

M Sivakumaran

Molecular

Haematology Unit,

Leeds General

Infirmary Leeds

M Short

N W Regional

Immunology Service,

St Mary's Hospital,

Manchester

R S H Pumphrey

Correspondence to:

Dr M Sivakumaran

DrM Sivakum

Department of

Hoyal Infirmary, Leicester Royal Infirmary.

Accepted for publication

9 February 1994

\section{$(\mathcal{F}$ Clin Pathol 1994;47:676-679)}

Pure red cell aplasia (PRCA) is characterised by normocytic anaemia, reticulocytopenia, and severe erythroid hypoplasia of the bone marrow associated with normal myeloid and megakaryocytic cell lines. ${ }^{1}$ It is divided into congenital and acquired categories, the latter being further subdivided into two subclasses-primary and secondary. About 50\% of patients with PRCA have a thymoma. Conversely, because PRCA is rare, it occurs in only $5 \%$ of patients with thymoma. ${ }^{2}$ There is also a well recognised association between thymomas and myasthenia gravis. However, the simultaneous occurrence of all three disorders is extremely rare. ${ }^{3}$ Some cases of socalled primary PRCA have large granular lymphocytic leukaemia. ${ }^{4}$ This is a relatively recently recognised condition characterised by a neoplastic proliferation of large granular lymphocytes.

\section{Case report}

A 73 year old man presented with a four week history of symptoms of anaemia. His blood count showed a haemoglobin of $41 \mathrm{~g} / 1$ with a mean corpuscular volume (MCV) of $100 \mathrm{fl}$, a mild lymphocytosis $\left(5 \cdot 7 \times 10^{9} / 1\right)$, and a normal platelet count. Serum vitamin B12 and folate concentrations were normal. A bone marrow aspirate confirmed a diagnosis of pure red cell aplasia. Although a chest radiograph showed changes of chronic airways disease only, a computed tomography scan of the thorax showed a soft tissue mass in the anterior mediastinum that was suspicious of thymoma. There was a polyclonal increase in globulins with raised IgG and IgM. A random blood glucose sample showed an increased concentration and a glucose tolerance test was consistent with diabetes mellitus. $\mathrm{He}$ was initially managed with blood transfusions.

A month after presentation, he developed a unilateral partial ptosis. $\mathrm{He}$ also admitted to intermittent diplopia, some leg weakness, and non-progressive dysphagia. On examination he had a left partial ptosis and bilateral shoulder abduction weakness on exercise. A tensilon test was positive. Antibodies to acetylcholine receptor were increased and he later developed antibodies to striated muscle. Myasthenia gravis was diagnosed and he was given Pyridostigmine.

While awaiting thymectomy, his peripheral blood lymphocyte count increased, reaching a peak of $12 \cdot 1 \times 10^{9} / 1$ of which about $30 \%$ were large granular lymphocytes. Immunophenotyping of the peripheral blood and bone marrow lymphocytes showed that about $70 \%$ were T cells ( $67 \%$ with $a \beta$ and $2 \%$ with $\gamma \delta \mathrm{T}$ cell antigen receptors). About $30 \%$ of the peripheral blood mononuclear cells were of the natural killer cell phenotype $(\mathrm{CD} 2+$, CD3-, CD7 +, CD16 +, CD57 + ). A monoclonal antibody panel containing seven antibodies against the variable regions of the TCR showed the peripheral blood $T$ lymphocytes to be polyclonal. Infection with HTLV 